\title{
MicroRNA-429 inhibits the migration and invasion of colon cancer cells by targeting PAK6/cofilin signaling
}

\author{
XIANGYANG TIAN $^{1,2}$, ZIBAI WEI $^{2}$, JIA WANG $^{4}$, PING LIU $^{2}$, YIJUN QIN $^{3}$ and MEIZUO ZHONG ${ }^{1}$ \\ ${ }^{1}$ Department of Oncology, Xiangya Hospital of Central South University, Changsha, Hunan 410008; \\ Departments of ${ }^{2}$ Oncology and ${ }^{3}$ Pathology, ${ }^{4}$ Immunology, Peace Hospital of Changzhi Medical College, \\ Changzhi, Shanxi 046000, P.R. China
}

Received January 22, 2015; Accepted April 24, 2015

DOI: $10.3892 / o r .2015 .4039$

\begin{abstract}
MicroRNAs (miRs), a class of non-coding RNAs 18-25 nucleotides in length, can lead to mRNA degradation or inhibit protein translation by directly binding to the 3'-untranslational region (UTR) of their target mRNAs. The deregulation of miR-429 has been suggested to be involved in the development and progression of colon cancer. However, the detailed molecular mechanism involved remains to be determined. The aim of the present study was to investigate the role of miR-429 in the regulation of migration and invasion of colon cancer cells using RT-qPCR and western blotting. The results showed that the expression of miR-429 was reduced in colon cancer cell lines, when compared to a normal colon epithelial cell line. Treatment with DNA demethylation agent 5-aza-2'-deoxycytidine and histone deacetylase inhibitor phenylbutyrate (PBA), or transfection with the pre-miR-429 lentivirus plasmid led to the upregulation of miR-429 expression, as well as inhibition of migration and invasion in colon cancer cells. Investigation of the molecular mechanism showed that PAK6 was a novel target of miR-429, and the expression of PAK6 was upregulated in colon cancer tissues and cell lines, and was negatively regulated by miR-429 in colon cancer cells. Moreover, the cofilin signaling acted as a downstream effector of miR-429 in colon cancer cells. In conclusion, the results of the present study suggested that miR-429 inhibits the migration and invasion of colon cancer cells, partly at least, by mediating the expression of PAK6, as well as the activity of cofilin signaling. Therefore, miR-429 is as a potential molecular target for the treatment of colon cancer.
\end{abstract}

\section{Introduction}

Colon cancer is one of the most common types of cancer. Although surgery combined with chemo- and radiotherapy,

Correspondence to: Professor Meizuo Zhong, Department of Oncology, Xiangya Hospital of Central South University, No. 87 Xiangya Road, Changsha, Hunan 410008, P.R. China

E-mail: doctorzhongmeizuo@163.com

Key words: colon cancer, microRNA-429, migration, invasion, PAK6, cofilin remains the main form of treatment, the median survival rate of colon cancer remains poor (1). As the development and progression of colon cancer are mediated by a complex cascade of molecular events, understanding of the molecular mechanism contributing to colon cancer is crucial (2).

MicroRNAs (miRs), a class of non-coding RNAs of 18-25 nucleotides in length, can lead to mRNA degradation or inhibit protein translation by directly binding to the 3'-untranslational region (UTR) of their target mRNAs (3). By directly mediating the expression levels of their target genes, miRs are closely associated with various biological processes, such as cell proliferation, cell cycle progression, survival, apoptosis, differentiation, motility as well as tumorigenesis (4). Deregulation of miRs is involved in the development and progression of various malignant tumors, including colon cancer (5).

Deregulation of miR-429 has been found to be involved in several types of cancer, such as non-small cell lung cancer, oropharyngeal carcinoma, pancreatic and colon cancer (6-10). Gaedcke et al (9) showed that miR-429 was downregulated in colon cancer tissues compared to normal rectal mucosa, suggesting that miR-429 is associated with the tumorigenesis of colon cancer. However, the exact role of miR-429 in the regulation of the migration and invasion of colon cancer cells, as well as the underlying molecular mechanisms involved remains unclear.

In the present study, we aimed to investigate the role of miR-429 in the regulation of migration and invasion of colon cancer cells. In addition, we explored the underlying molecular mechanisms.

\section{Materials and methods}

Reagents and materials. Dulbecco's modified Eagle's medium (DMEM), fetal bovine serum (FBS), TaqMan MicroRNA assay kit, BCA protein assay kit, ECL western blotting kit and Lipofectamine 2000 were purchased from Life Technologies (Carlsbad, CA, USA). Demethylation drug 5-aza-2'-deoxycytidine (5-Aza) and histone deacetylase inhibitor 4-phenylbutyric acid (PBA) were purchased from Sigma (St. Louis, MO, USA). A miRNeasy Mini kit was purchased from Qiagen (Valencia, CA, USA). The mouse anti-human antibodies and rabbit antimouse secondary antibody were purchased from Immunoway 
(Cambridge, UK). The Cell Invasion assay kit was purchased from Merck Millipore (Darmstadt, Germany).

Cell culture. Human SW620 and LOVO colon cancer cell lines, and a normal HCEpiC colon epithelium cell line were obtained from the Cell Bank of Central South University, Changsha, China. The cells were cultured in DMEM supplemented with $10 \% \mathrm{FBS}$ in a humidified atmosphere containing $5 \% \mathrm{CO}_{2}$ at $37^{\circ} \mathrm{C}$.

Tissue microarray immunohistochemistry. The tissue microarray (Auragene Bioscience Co., Changsha, China) covering 39 colon cancer tissues and their matched adjacent normal tissues was prepared for immunohistochemistry. Tissue microarray blocks were incubated with anti-PAK6 antibody or normal rabbit $\operatorname{IgG}$ as the negative control. Immunohistochemical staining was performed using the Moticam 3000 system with diaminobenzidine (Zhongshan Jinqiao Co., Beijing, China). Image-Pro Plus software was used to quantify the mean density of PAK6 staining, according to the manufacturer's instructions.

$R T-q P C R$. miRNAs were isolated from cells using the miRNeasy Mini kit according to the manufacturer's instructions. miRNA expression was then determined using the TaqMan MicroRNA assay kit on a 7500 Fast Real-Time PCR System (Applied Biosystems, Carlsbad, CA, USA), in according to the manufacturer's instructions. U6 was used as an endogenous reference. For each sample, independent experiments were repeated three times. The relative expression of miRNA was analyzed using the $2^{-\Delta \Delta C t}$ method.

Epigenetic cell drug treatment. Human SW620 and LOVO colon cancer cells were treated with 5-Aza $(15.55 \mathrm{nM})$ and PBA $(1.5 \mathrm{nM})$ for $72 \mathrm{~h}$.

Luciferase assay. Luciferase assay was performed to determine whether PAK6 was the direct target of miR-429. A wild-type or a mutant type of PAK6 3'-UTR was subcloned into the psiCHECK $^{\mathrm{TM}} 2$ luciferase miRNA expression reporter vector. The psiCHECK-PAK6-3'-UTR or psiCHECK2-mut PAK6-3'-UTR vector plus miR-429 or miR-429 inhibitors were cotransfected into colon cancer LOVO cells.

Western blot analysis. The cells were lysed in cold RIPA buffer. The BCA protein assay kit was used to determine the protein concentration. Protein was then separated with $10 \%$ SDS-PAGE, and transferred to a PVDF membrane. The PVDF membrane was blocked in 5\% non-fat dried milk in PBS for $4 \mathrm{~h}$. Subsequently, the PVDF membrane was incubated with specific primary antibodies for $3 \mathrm{~h}$. After being washed with PBS three times, each time for $5 \mathrm{~min}$, the PVDF membrane was incubated with the appropriate secondary antibody. After being washed with PBS three times, each time for $5 \mathrm{~min}$, an ECL western blotting kit was used to detect the immune complexes on the PVDF membrane.

Wound-healing assay. A wound-healing assay was performed to evaluate the cell migratory ability. Briefly, the cells were cultured to full confluence. Wounds of $\sim 1 \mathrm{~mm}$ width were created with a plastic scriber, and the cells were washed and

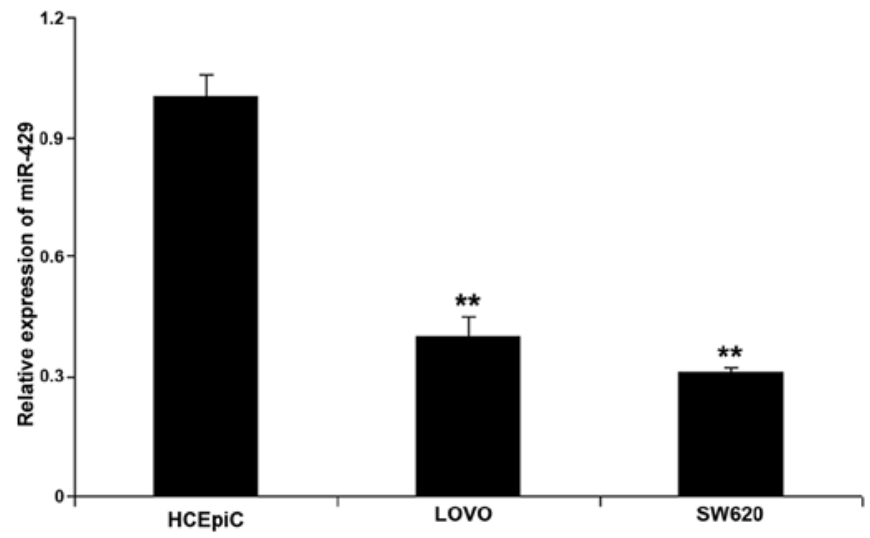

Figure 1. Quantitative PCR was performed to determine the relative expression of miR-429 in SW620 and LOVO colon cancer cell lines, and the HCEpiC colon epithelial cell line. ${ }^{* *} \mathrm{P}<0.01$ vs. HCEpiC.

incubated in a serum-free medium. After wounding for $24 \mathrm{~h}$, the cells were incubated in a medium including $10 \%$ FBS. Cultures at 0 and $48 \mathrm{~h}$ were fixed and observed under a microscope.

Transwell cell invasion assay. Cells treated with the indicated drugs for $72 \mathrm{~h}$ were starved in serum-free medium for $24 \mathrm{~h}$, and then resuspended in serum-free medium. The cells were added to the upper chamber, while the lower chamber was filled with base medium containing $10 \%$ FBS. After incubation for $24 \mathrm{~h}$, the cells attached to the bottom were stained with crystal violet for $20 \mathrm{~min}$, washed and air dried. Invasive cells were observed under a microscope.

Statistical analysis. Data were presented as mean \pm SD of three independent experiments. Statistical analysis was performed using SPSS 17.0 statistical software (SPSS, Inc., Chicago, IL, USA). The differences between groups were determined using the one-way ANOVA. $\mathrm{P}<0.05$ was considered to indicate a statistically significant result.

\section{Results}

miR-429 is downregulated in colon cancer cells. We first examined the expression level of miR-429 in the SW620 and LOVO colon cancer cell lines, and normal HCEpiC colon epithelial cells. The expression level of miR-429 was significantly reduced in the SW620 and LOVO colon cancer cell lines, compared to the normal HCEpiC colon epithelial cells (Fig. 1).

Upregulation of miR-429 inhibits the migration and invasion of colon cancer cells. After treatment with 5-Aza and PBA in SW620 and LOVO colon cancer cells, we determined the expression level of miR-429. As shown in Fig. 2A, miR-429 was significantly upregulated following treatment with 5-Aza and PBA. We also transfected the colon cancer cells with pre-miR-429 plasmid, and showed that the expression of miR-429 was increased after transfection (Fig. 2B). We then investigated the effect of miR-429 upregulation on colon cancer cell migration and invasion. The results showed that the upregulation of miR-429 markedly suppressed colon cancer cell migration (Fig. 3). We also found that the upregulation 

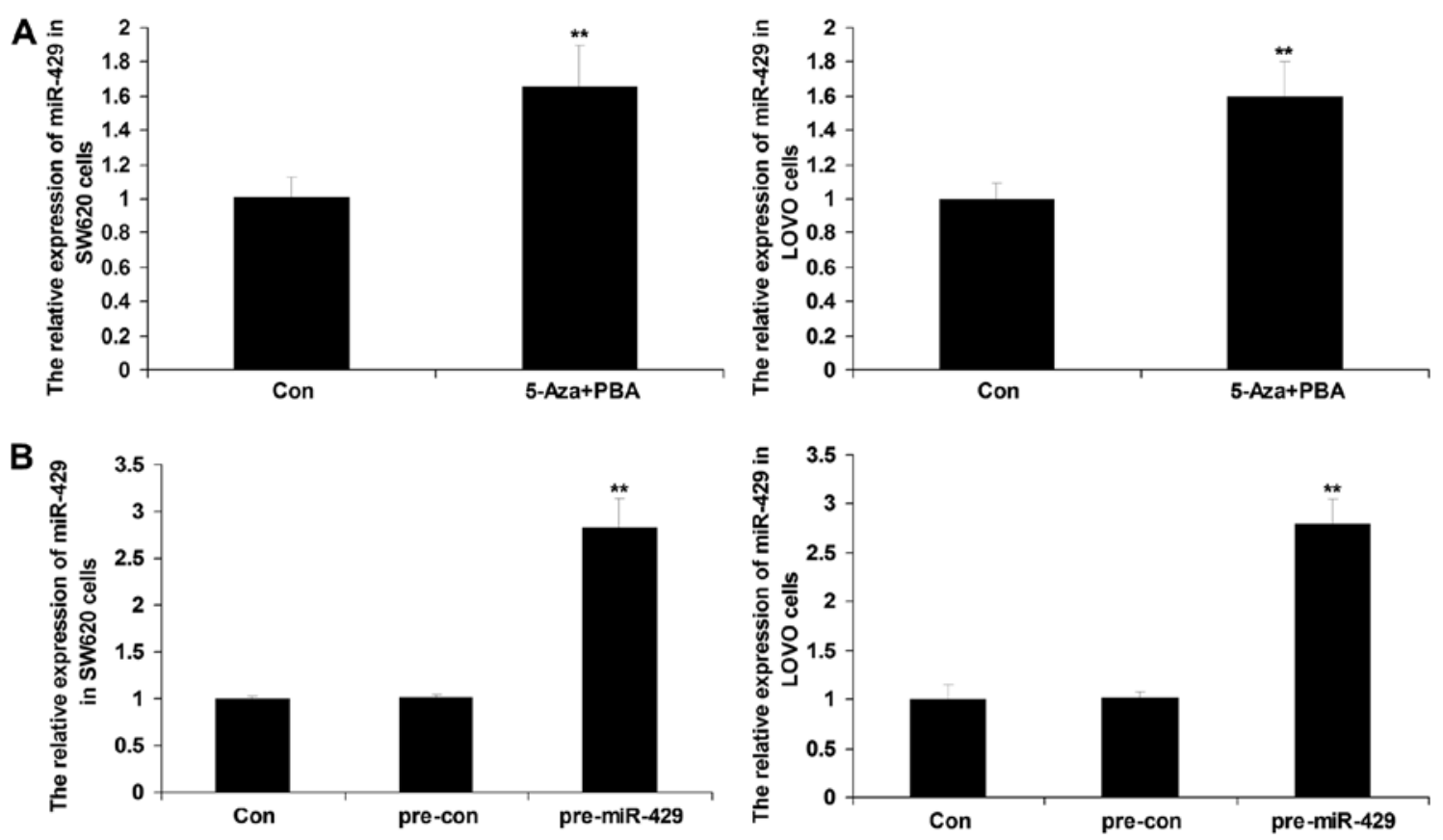

Figure 2. (A) Quantitative PCR was performed to determine the relative expression of miR-429 in SW620 and LOVO colon cancer cells treated with 5-Aza $(15.55 \mathrm{nM})+$ PBA $(1.5 \mathrm{nM})$. Con, SW620 and LOVO cells without any treatment. ${ }^{* *} \mathrm{P}<0.01$ vs. Con. (B) Quantitative PCR was performed to determine the relative expression of miR-429 in colon cancer SW620 and LOVO cells transfected with pre-con plasmid (as a control) or pre-miR-429 plasmid, respectively. Con, cells without any treatment. ${ }^{* *} \mathrm{P}<0.01$ vs. Con.

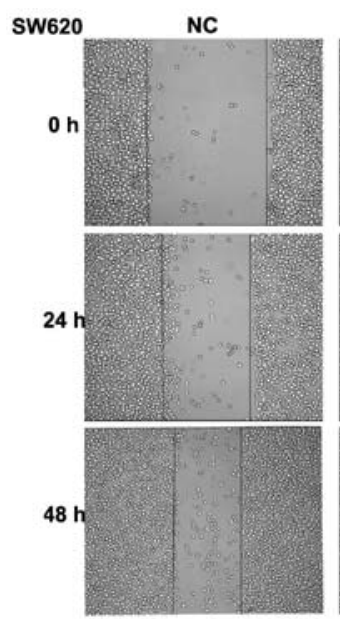

Lovo

NC
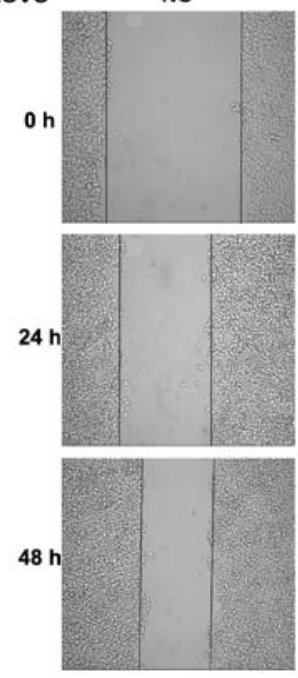

5-Aza+PBA
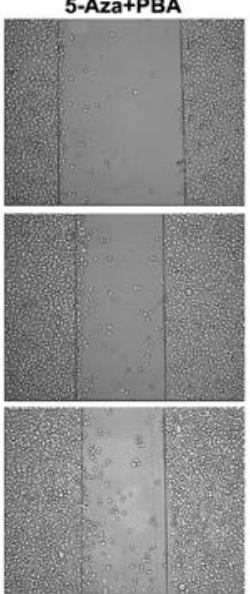

5-Aza+PBA
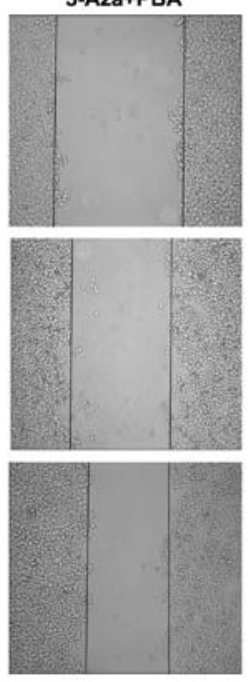

Lv-pre-con
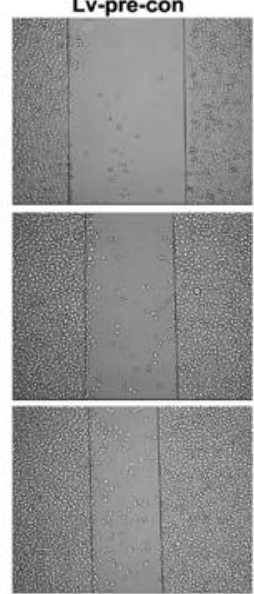

Lv-pre-con
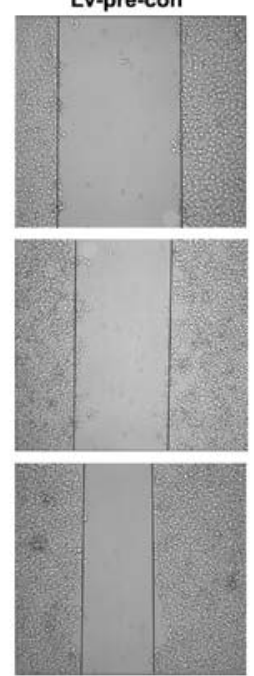
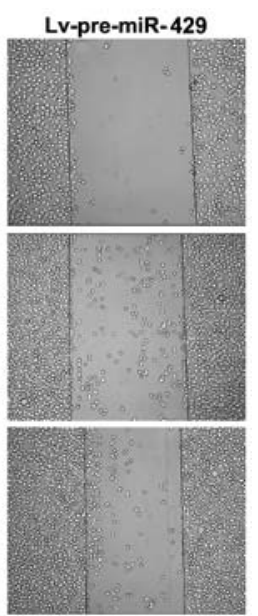

Lv-pre-miR-429
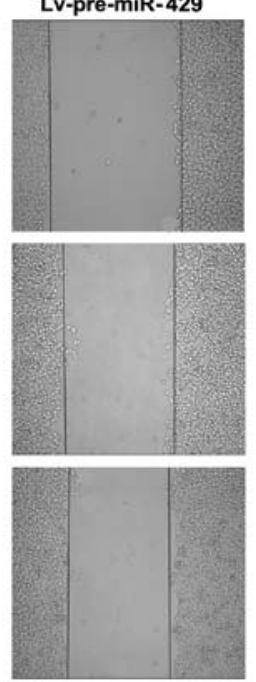

Figure 3. A wound-healing assay was performed to determine the migratory ability of colon cancer SW620 and LOVO cells treated with 5-Aza (15.55 nM) + PBA (1.5 nM), or transfected with pre-con plasmid (as a control) or pre-miR-429 plasmid, respectively. NC, cells without any treatment or transfection. 

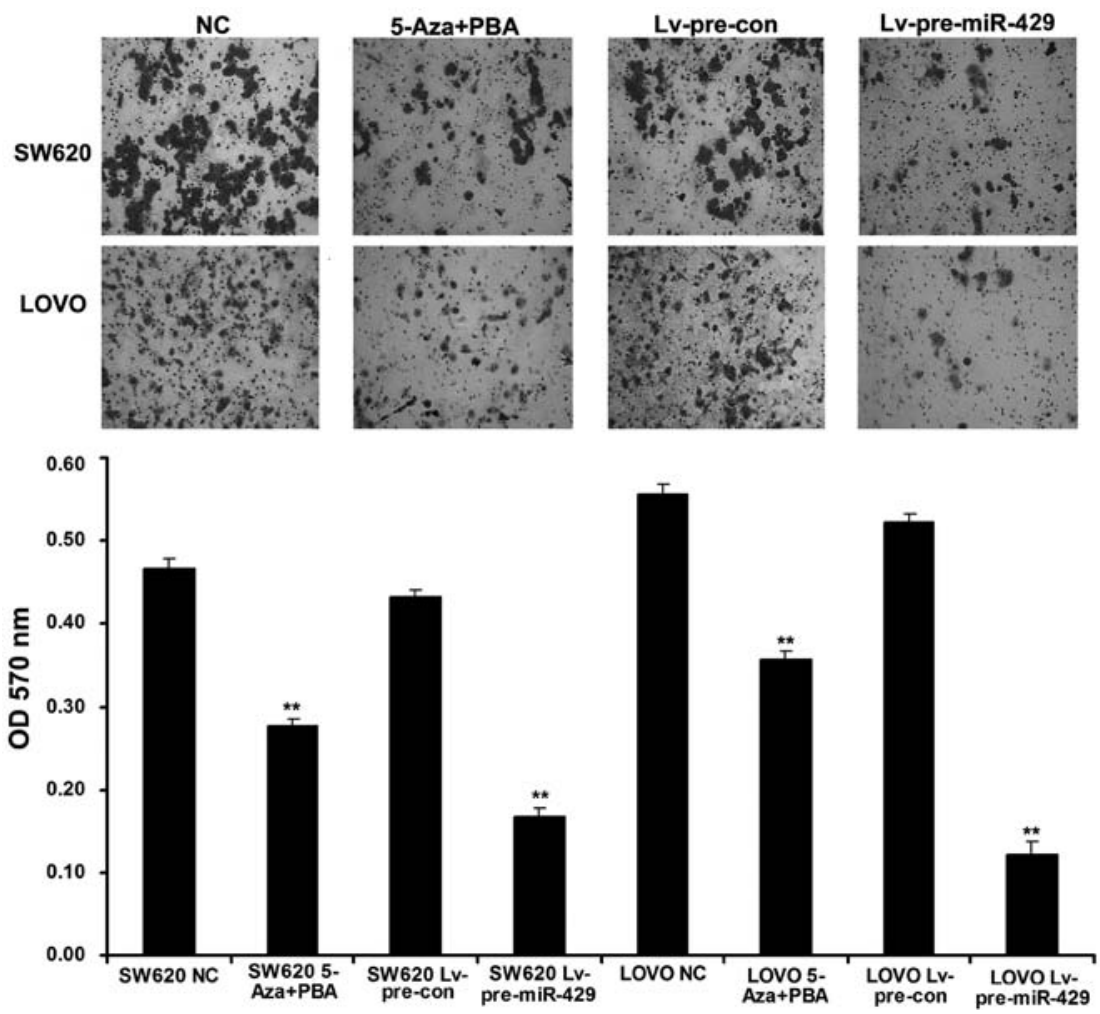

Figure 4. A Transwell assay was performed to determine the invasive ability of SW620 and LOVO colon cancer cells treated with 5-Aza (15.55 nM) + PBA $(1.5 \mathrm{nM})$, or transfected with pre-con plasmid (as a control) or pre-miR-429 plasmid, respectively. NC, cells without any treatment or transfection. ${ }^{* *} \mathrm{P}<0.01 \mathrm{vs} . \mathrm{NC}$.
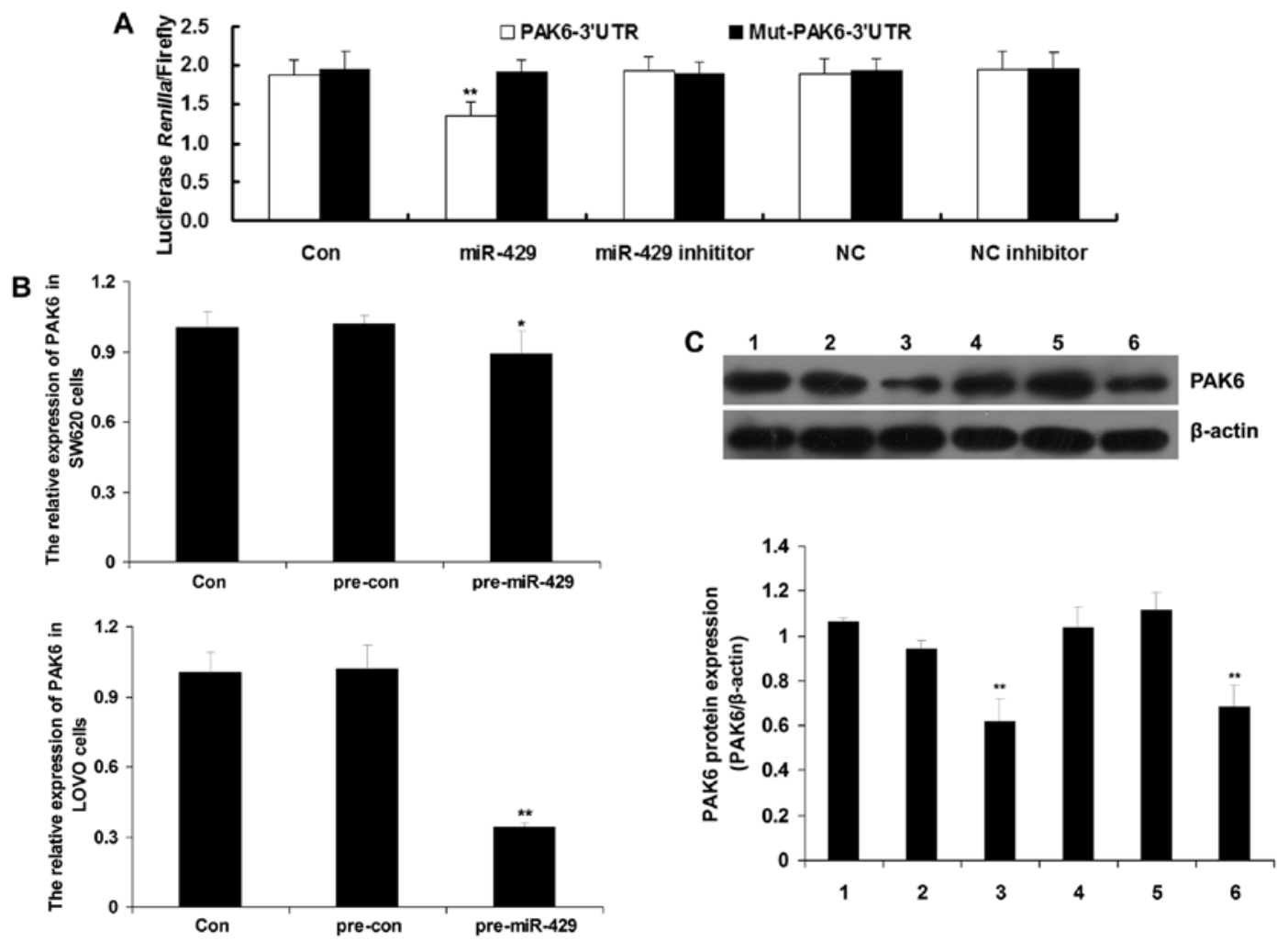

Figure 5. PAK6 is a target gene of miR-429. (A) Luciferase assay was performed to determine whether PAK6 was the direct target of miR-429. A wild-type or a mutant type of PAK6 3'-UTR was subcloned into the psiCHECK ${ }^{\mathrm{TM}} 2$ luciferase miRNA expression reporter vector, respectively. PsiCHECK ${ }^{\mathrm{TM}}$-PAK6-3'-UTR or psiCHECK ${ }^{\mathrm{TM}}$ 2-mut PAK6-3'-UTR vector plus miR-429 or miR-429 inhibitor were co-transfected into LOVO colon cancer cells. Con, LOVO cells without any transfection; NC, cells transfected with NC virus; NC inhibitor, cells transfected with NC inhibitor. ${ }^{* *} \mathrm{P}<0.01$ vs. Con. Quantitative PCR was performed to determine the relative mRNA expression of PAK6 in SW620 and LOVO colon cancer cells transfected with pre-con plasmid (as a control) or pre-miR-429 plasmid, respectively. Con, cells without any treatment. ${ }^{*} \mathrm{P}<0.05$ vs. Con. ${ }^{* *} \mathrm{P}<0.01$ vs. Con. (C) Western blot assay was performed to determine the relative protein expression of PAK6 in colon cancer LOVO (1-3) and SW620 (4-6) cells transfected with pre-con plasmid (2 and 5) or pre-miR-429 plasmid (3 and 6), respectively. Lanes 1 and 4 , cells without any treatment as control. ${ }^{* *} \mathrm{P}<0.01$ vs. Con. 


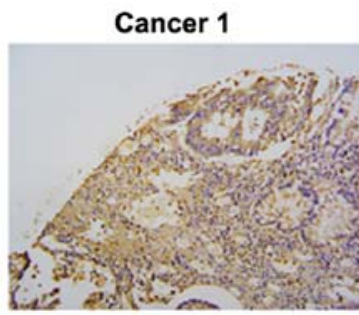

Cancer 3

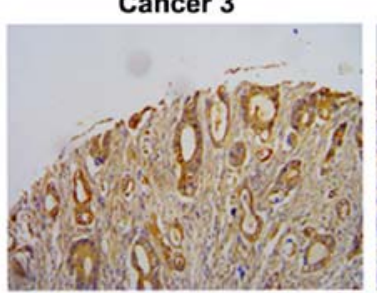

Adjacent 1

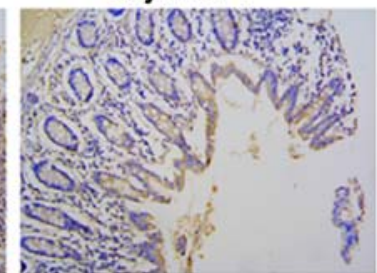

Adjacent 3

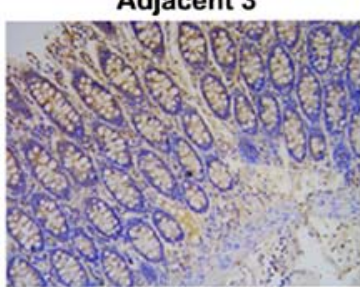

Cancer 2

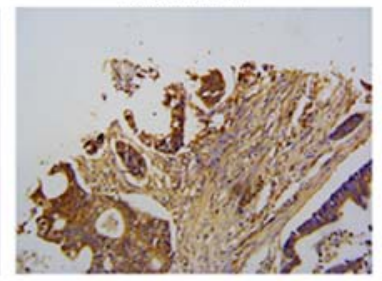

Cancer 4

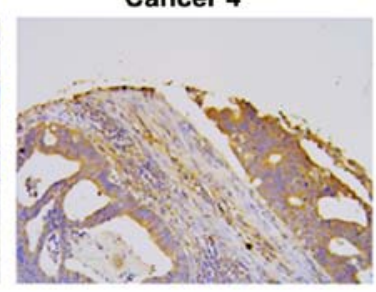

Adjacent 2

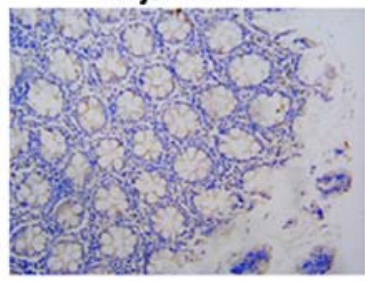

Adjacent 4

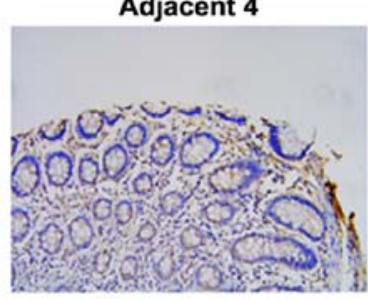

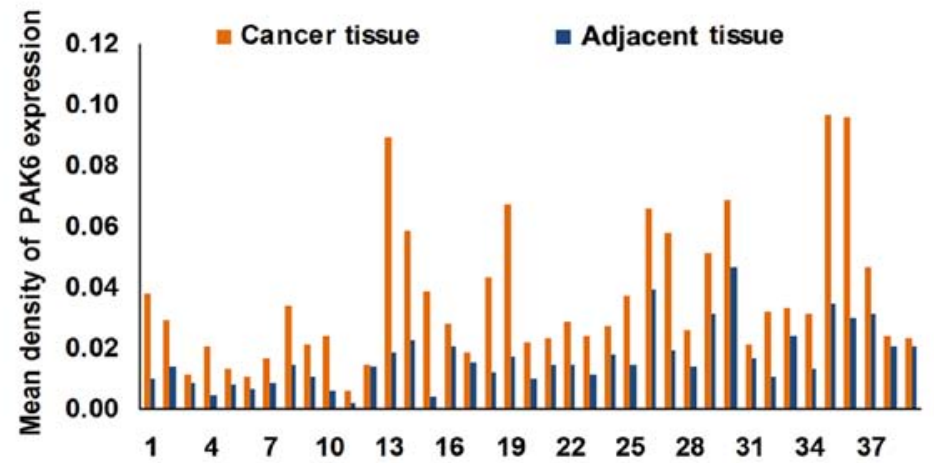

Figure 6. Tissue microarray immunohistochemistry was performed to detect the expression of PAK6 in colon cancer tissues as well as their matched adjacent normal tissues. Four representative pairs are shown.

of miR-429 markedly suppressed colon cancer cell invasion (Fig. 4). These results suggested that miR-429 plays a suppressive role in colon cancer metastasis.

PAK6 is identified as a target gene of miR-429 in colon cancer cells. We focused on the targets of miR-429 in colon cancer cells, and the luciferase reporter assay data confirmed that PAK6 was a target gene of miR-429 in LOVO colon cancer cells (Fig. 5A). We also found that transfection with pre-miR-429 led to a decreased mRNA and protein expression of PAK6 (Fig. 5B and C), suggesting that miR-429 negatively mediated the expression of PAK6 in colon cancer cells.

PAK6 expression is upregulated in colon cancer tissues. We determined the expression of PAK6 in colon cancer tissues as well as their matched adjacent normal tissues. As shown in Fig. 6, the expression of PAK6 was positive in colon cancer tissues, but negative in their matched adjacent normal tissues, suggesting that PAK6 acts as an oncogene in colon cancer.

Cofilin acts a downstream effector in miR-429-mediated colon cancer cell migration and invasion. It has been established that cofilin plays a role in the regulation of cell motility (11). Accordingly, we investigated whether cofilin was involved in miR-429-mediated migration and invasion of SW620 and LOVO colon cancer cells. The antibody of p-cofilin was used to inhibit the activity of cofilin. As shown in Fig. 7, inhibition of cofilin activity markedly suppressed the migration of colon cancer cells, and enhanced the inhibitory effect of miR-429 upregulation on colon cancer cell migration. Similar results were observed in the cell invasion assay. As shown in Fig. 8, inhibition of cofilin activity also suppressed the invasion of colon cancer cells, even in colon cancer cells transfected with anti-miR-429. Taken together, the results suggested that cofilin acts a downstream effector in miR-429-mediated colon cancer cell migration and invasion.

\section{Discussion}

The results of the present study have shown that the expression level of miR-429 was significantly downregulated in colon cancer cells, when compared to normal colon epithelial cells. Treatment with epigenetic drugs or pre-miR-429 lentivirus plasmid led to the upregulation of miR-429 expression, which further resulted in the decreased migration and invasion of colon cancer cells. PAK6, upregulated in colon cancer tissues and cells, was also identified as a target gene of miR-429, and the expression of PAK6 was negatively regulated by miR-429 in colon cancer cells. Investigation of the molecular mechanism showed that PAK6/cofilin signaling was involved in miR-429-mediated colon cancer cell migration and invasion.

The exact role of miR-429 in colon cancer remains to be clarified. Previous findings have demonstrated that the dysfunction of miR-429 is involved in the development and 


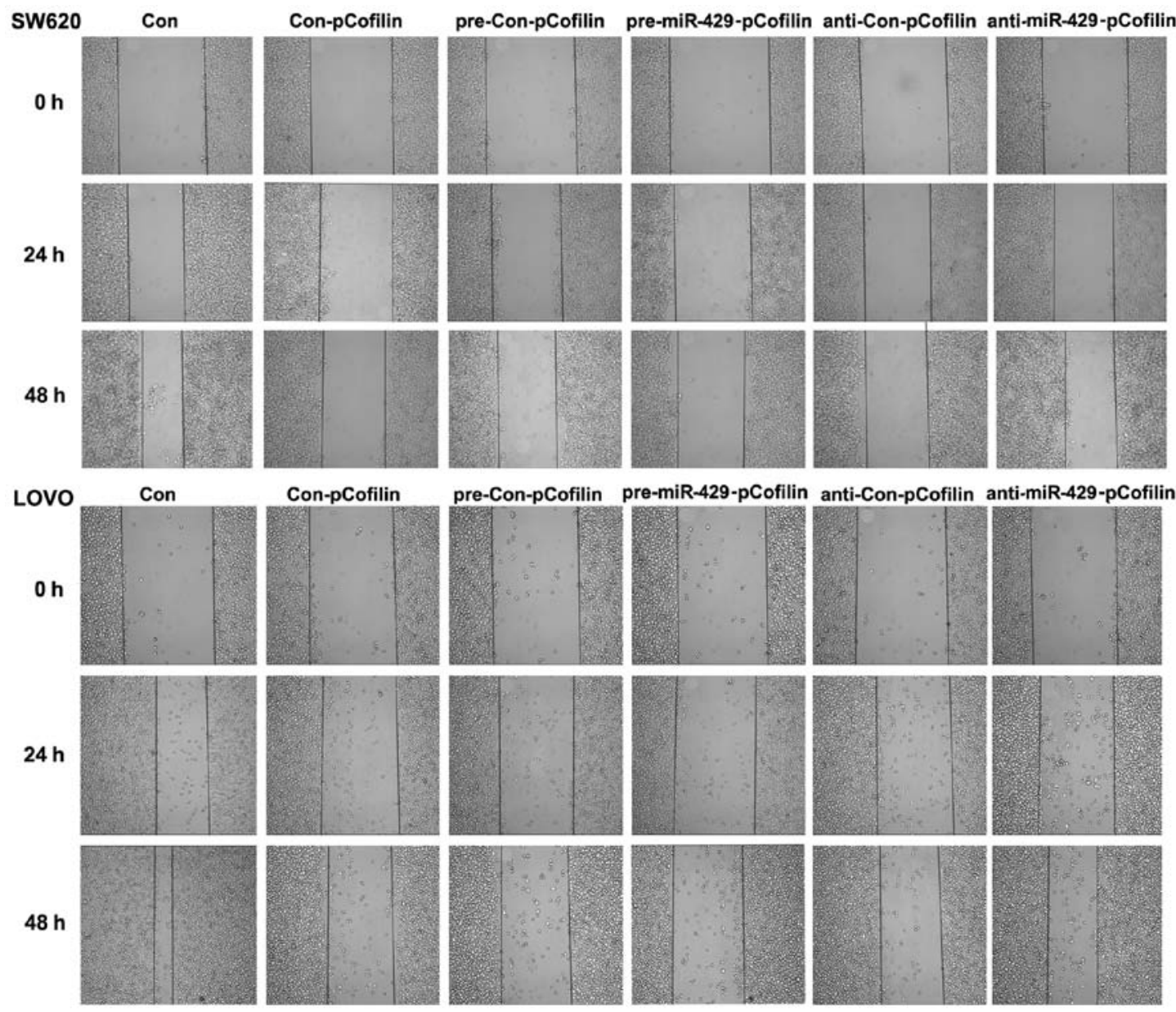

Figure 7. Scratch assay was performed to determine the migratory capacity of colon cancer LOVO and SW620 cells transfected with control p-cofilin antibody (con-p-cofilin), control pre-miRNA (pre-Con)+p-cofilin antibody, pre-miR-429+p-cofilin antibody, control anti-miRNA (anti-Con)+p-cofilin antibody, anti$429+$ p-cofilin antibody, respectively. Con, cells without any transfection.
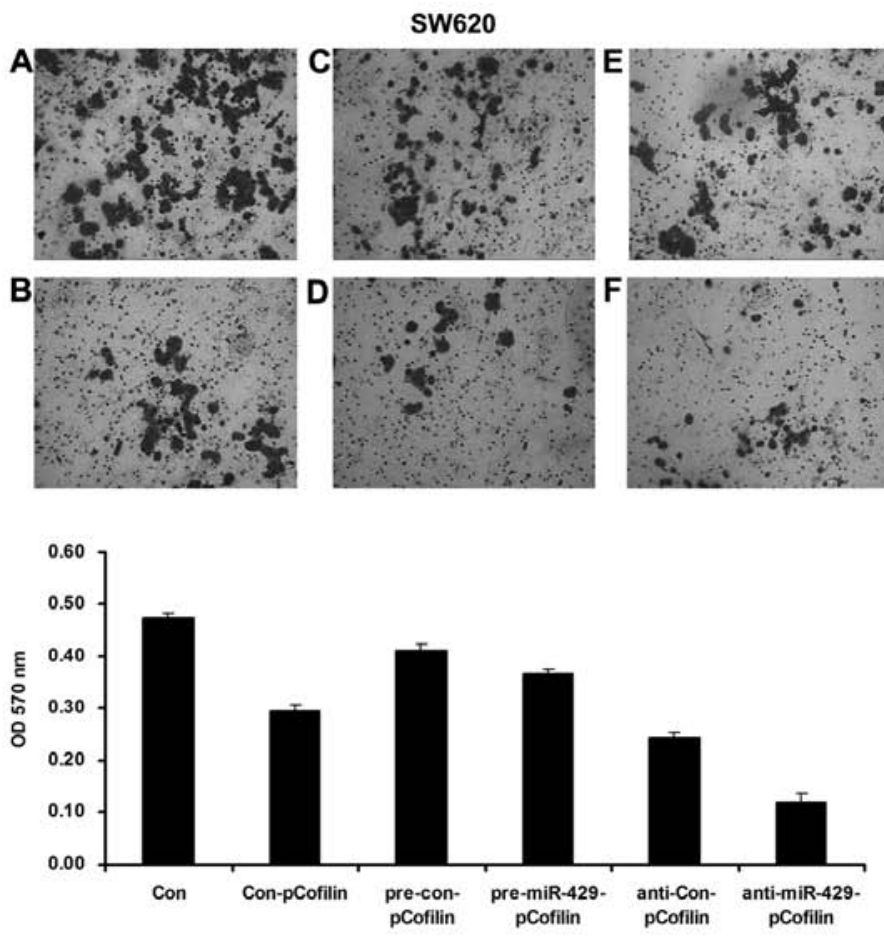
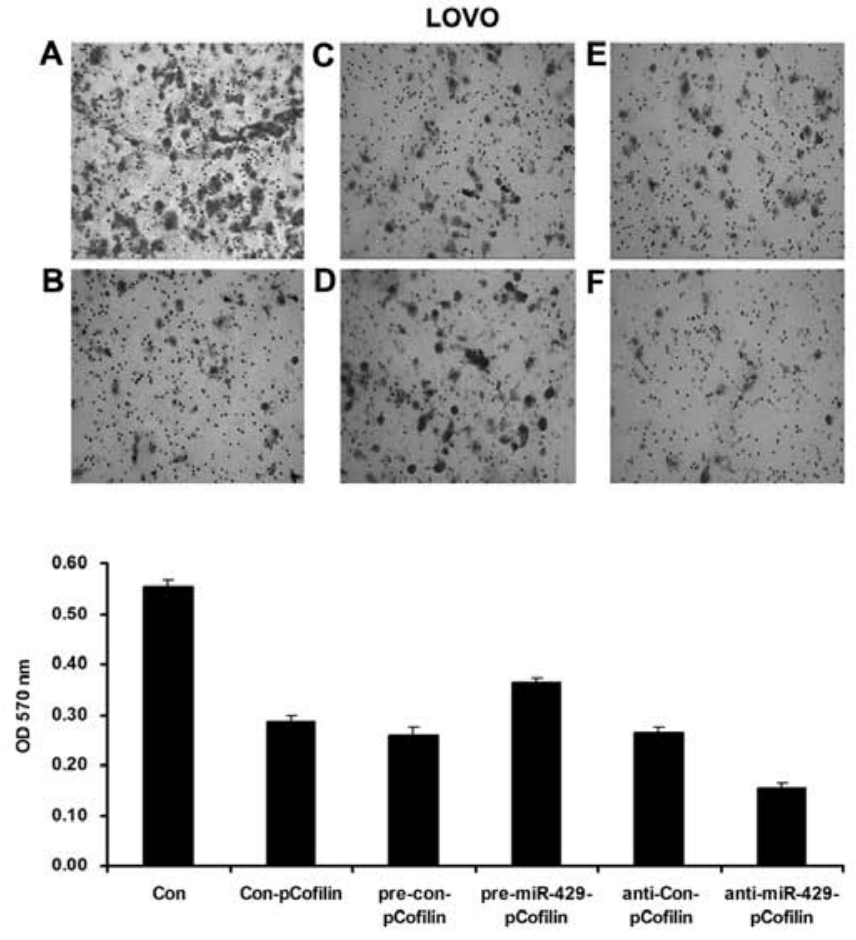

Figure 8. Transwell assay was performed to determine the invasive capacity of colon cancer LOVO and SW620 cells transfected with control p-cofilin antibody (B), control pre-miRNA (pre-Con)+p-cofilin antibody (C), pre-miR-429+p-cofilin antibody (D), control anti-miRNA (anti-Con)+p-cofilin antibody (E), anti$429+$ p-cofilin antibody $(\mathrm{F})$, respectively. Con $(\mathrm{A})$, cells without any transfection. 
progression of many malignant tumors (12). For instance, miR-429 has been suggested to participate in the tumorigenesis of non-small cell lung cancer, retinoblastoma, pancreatic cancer and oropharyngeal carcinoma $(6-8,10)$. Recently, the expression level of miR-429 was found to be significantly reduced in colon cancer tissues (9). In the present study, we showed that miR-429 was markedly downregulated in colon cancer cell lines, when compared with that in normal colon epithelial cells. These findings suggest that miR-429 potentially plays a role in colon cancer.

It has been well established that DNA methylation and histone acetylation act as key regulators in miRNA transcription. Generally, DNA demethylation and histone acetylation play promoting roles in gene transcription (13). However, whether DNA methylation and histone acetylation are involved in the regulation of miR-429 in cancer cells has not been previously investigated. To confirm that DNA methylation and histone acetylation are associated with the downregulation of miR-429 in colon cancer cells, we SW620 and LOVO cells with 5-Aza and PBA. 5-Aza is a DNA methyltransferase inhibitor that leads to DNA demethylation (14). PBA is a histone deacetylase inhibitor that leads to histone acetylation (15). Our results show that treatment with 5-Aza and PBA significantly upregulated the expression level of miR-429 in colon cancer cells, indicating that the expression level of miR-429 in colon cancers was closely regulated by epigenetic modulations. Upregulation of miR-429 led to decreased migratory and invasive abilities of colon cancer cells, suggesting that miR-429 plays an inhibitory role in the regulation of colon cancer metastasis.

PAK6 belongs to a family of p21-stimulated serine/threonine protein kinases that is associated with several cell processes, including apoptosis, cytoskeletal rearrangement, as well as the mitogen-activated protein (MAP) kinase signaling pathway (16-19). Moreover, PAK6 can interact with the androgen receptor and translocate to the nucleus, and plays a role in the regulation of gene transcription $(20,21)$. The deregulated expression of PAK6 has been identified in several cancers, including prostate cancer, hepatocellular carcinoma and thyroid cancer (22-24). In the present study, we identified, to the best of our knowledge, for the first time PAK6 is a direct target gene of miR-429 in colon cancer cells and that PAK6, upregulated in colon cancer cells, was negatively regulated by miR-429. As PAK6 has been demonstrated to be associated with cancer cell metastasis (25), we suggest that the suppressive effect of miR-429 upregulation on colon cancer cell migration and invasion may occur, partly at least, by directly targeting PAK6. Moreover, investigation of the molecular mechanism revealed that cofilin signaling was involved in the miR-429-mediated migration and invasion in colon cancer cells. It has been demonstrated that cofilin promotes invadopodial membrane recycling during cell invasion (11). Moreover, cofilin has been demonstrated to act as a key regulator in cancer cell motility $(26,27)$. For instance, Chen et al (28) showed that cofilin was a critical signaling pathway involved in miR-138-mediated ovarian cancer cell metastasis. Accordingly, we suggest that miR-429 played an inhibitory role in the regulation of colon cancer cell migration and invasion, partially at least, through the modulation of PAK6 as well as cofilin.
In summary, miR-429 may serve as a potential agent for the treatment of colon cancer.

\section{References}

1. Khare S and Verma M: Epigenetics of colon cancer. Methods Mol Biol 863: 177-185, 2012.

2. Rajamanickam S and Agarwal R: Natural products and colon cancer: Current status and future prospects. Drug Dev Res 69: 460-471, 2008.

3. Bartel DP: MicroRNAs: Genomics, biogenesis, mechanism, and function. Cell 116: 281-297, 2004.

4. Ambros V: The functions of animal microRNAs. Nature 431: 350-355, 2004.

5. Baer C, Claus R and Plass C: Genome-wide epigenetic regulation of miRNAs in cancer. Cancer Res 73: 473-477, 2013.

6. Hui AB, Lin A, Xu W, Waldron L, Perez-Ordonez B, Weinreb I, Shi W, Bruce J, Huang SH, O'Sullivan B, et al: Potentially prognostic miRNAs in HPV-associated oropharyngeal carcinoma. Clin Cancer Res 19: 2154-2162, 2013.

7. Yoon KA, Yoon H, Park S, Jang HJ, Zo JI, Lee HS and Lee JS: The prognostic impact of microRNA sequence polymorphisms on the recurrence of patients with completely resected non-small cell lung cancer. J Thorac Cardiovasc Surg 144: 794-807, 2012.

8. Zhao JJ, Yang J, Lin J, Yao N, Zhu Y, Zheng J, Xu J, Cheng JQ, Lin JY and Ma X: Identification of miRNAs associated with tumorigenesis of retinoblastoma by miRNA microarray analysis. Childs Nerv Syst 25: 13-20, 2009.

9. Gaedcke J, Grade M, Camps J, Sokilde R, Kaczkowski B, Schetter AJ, Difilippantonio MJ, Harris CC, Ghadimi BM, Moller S, et al: The rectal cancer microRNAome - microRNA expression in rectal cancer and matched normal mucosa. Clin Cancer Res 18: 4919-4930, 2012.

10. Schultz NA, Werner J, Willenbrock H, Roslind A, Giese N, Horn T, Wøjdemann M and Johansen JS: MicroRNA expression profiles associated with pancreatic adenocarcinoma and ampullary adenocarcinoma. Mod Pathol 25: 1609-1622, 2012.

11. Hagedorn EJ, Kelley LC, Naegeli KM, Wang Z, Chi Q and Sherwood DR: ADF/cofilin promotes invadopodial membrane recycling during cell invasion in vivo. J Cell Biol 204: 1209-1218, 2014.

12. Chen Y, Zhang Z, Yang K, Du J, Xu Y and Liu S: Myeloid zincfinger $1(\mathrm{MZF}-1)$ suppresses prostate tumor growth through enforcing ferroportin-conducted iron egress. Oncogene: Oct 6 , 2014. doi: 10.1038/onc.2014.310 (Epub ahead of print).

13. Boland MJ, Nazor KL and Loring JF: Epigenetic regulation of pluripotency and differentiation. Circ Res 115: 311-324, 2014.

14. Takaoka K, Hangaishi A, Ito A, Morioka T, Kida M and Usuki K: Late hematological improvement of myelodysplastic syndrome following treatment with 5-azacitidine therapy. Intern Med 53: 2241-2243, 2014

15. Daosukho C, Chen Y, Noel T, Sompol P, Nithipongvanitch R, Velez JM, Oberley TD and St Clair DK: Phenylbutyrate, a histone deacetylase inhibitor, protects against adriamycininduced cardiac injury. Free Radic Biol Med 42: 1818-1825, 2007.

16. Shepelev MV and Korobko IV: Pak6 protein kinase is a novel effector of an atypical Rho family GTPase Chp/RhoV. Biochemistry (Mosc) 77: 26-32, 2012.

17. Lee SH, Eom M, Lee SJ, Kim S, Park HJ and Park D: BetaPixenhanced p38 activation by $\mathrm{Cdc} 42 / \mathrm{Rac} / \mathrm{PAK} / \mathrm{MKK} 3 / 6$-mediated pathway. Implication in the regulation of membrane ruffling. $\mathrm{J}$ Biol Chem 276: 25066-25072, 2001.

18. Lee SR, Ramos SM, Ko A, Masiello D, Swanson KD, Lu ML and Balk SP: AR and ER interaction with a p21-activated kinase (PAK6). Mol Endocrinol 16: 85-99, 2002.

19. Jaffer ZM and Chernoff J: p21-activated kinases: Three more join the Pak. Int J Biochem Cell Biol 34: 713-717, 2002.

20. Yang F,Li X, Sharma M,Zarnegar M,Lim B and Sun Z: Androgen receptor specifically interacts with a novel p21-activated kinase, PAK6. J Biol Chem 276: 15345-15353, 2001.

21. Liu X, Busby J, John C, Wei J, Yuan X and Lu ML: Direct interaction between AR and PAK6 in androgen-stimulated PAK6 activation. PLoS One 8: e77367, 2013. 
22. Zapatero A, Morente M, Nieto S, Martín de Vidales C, Lopez C, Adrados M, Arellano R, Artiga MJ, Garcia-Vicente F, Herranz LM, et al: Predictive value of PAK6 and PSMB4 expression in patients with localized prostate cancer treated with dose-escalation radiation therapy and androgen deprivation therapy. Urol Oncol 32: 1327-1332, 2014.

23. Chen H, Miao J, Li H, Wang C, Li J, Zhu Y, Wang J, Wu X and Qiao H: Expression and prognostic significance of p21-activated kinase 6 in hepatocellular carcinoma. J Surg Res 189: 81-88, 2014.

24. McCarty SK, Saji M, Zhang X, Jarjoura D, Fusco A, Vasko VV and Ringel MD: Group I p21-activated kinases regulate thyroid cancer cell migration and are overexpressed and activated in thyroid cancer invasion. Endocr Relat Cancer 17: 989-999, 2010 .
25. Liu W, Liu H, Liu Y, Xu L, Zhang W, Zhu Y, Xu J and Gu J: Prognostic significance of p21-activated kinase 6 expression in patients with clear cell renal cell carcinoma. Ann Surg Oncol 21: 575-583, 2014.

26. Li H, Zhang B, Liu Y and Yin C: EBP50 inhibits the migration and invasion of human breast cancer cells via LIMK/cofilin and the PI3K/Akt/mTOR/MMP signaling pathway. Med Oncol 31: $162,2014$.

27. Huang X, Sun D, Pan Q, Wen W, Chen Y, Xin X, Huang M, Ding J and Geng M: JG6, a novel marine-derived oligosaccharide, suppresses breast cancer metastasis via binding to cofilin. Oncotarget 5: 3568-3578, 2014.

28. Chen P, Zeng M, Zhao Y and Fang X: Upregulation of Limk1 caused by microRNA-138 loss aggravates the metastasis of ovarian cancer by activation of Limk1/cofilin signaling. Oncol Rep 32: 2070-2076, 2014. 